\title{
Modelagem de Custo Total de Propriedade (TCO) de uma Infraestrutura Computacional em Nuvem
}

\author{
Igor W. S. Falcão ${ }^{1}$,Paulo H. A. Pereira ${ }^{1}$, Rafael F. Vieira ${ }^{1}$, Antonio C. Oliveira Jr ${ }^{2}$ \\ Daniel S. Souza ${ }^{1}$, Marcos C. R. Seruffo ${ }^{1}$, Diego L. Cardoso ${ }^{1}$. \\ ${ }^{1}$ Instituto de Tecnologia - Uinversidade Federal do Pará (UFPA) \\ Caixa Postal 8619 - 66.075-900 - Belém - PA - Brasil. \\ ${ }^{2}$ Instituto de Informática (INF) \\ Universidade Federal de Goiás (UFG) - Goiânia - GO - Brasil. \\ \{igorufpa2013.4, paulohenrique99.10, fogarollirafael\}@gmail.com, \\ \{antonio@inf.ufg.br\}, \\ \{danielssouza, seruffo, diego\}@ufpa.br
}

\begin{abstract}
Cloud computing, in addition to promoting a shared data storage and processing model, strengthens the Cloud Service Provider (CSP) technical and economic potential, presenting numerous investment possibilities. This paper proposes a cost model and an evaluation scenario based on Total Cost of Ownership (TCO), evaluating the context of acquisition and operation of equipment for the implementation of cloud infrastructure. Hence, it was possible to specify the main assets of typical cloud infrastructures and, above all, to evaluate their financial performance based on cost projections that took into account aspects that directly infer the return of Data Center investments.
\end{abstract}

Resumo. A computação em nuvem, além de promover um modelo de armazenamento e processamento de dados compartilhados, fortalece o potencial técnicoeconômico do CSP (Cloud Service Provider), apresentando inúmeras possibilidades no âmbito computacional. Neste trabalho, é proposto um modelo de custo e um cenário de avaliação com base no TCO (Total Cost of Ownership), avaliando o contexto, aquisição e operação de equipamentos para implantação de infraestruturas em nuvem. Dessa forma, foi possivel especificar os principais ativos de infraestruturas típicas de nuvem e sobretudo, avaliar seu desempenho financeiro a partir de projeções de custos considerando aspectos que inferem diretamente no retorno de investimentos de um Data Center.

\section{Introdução}

A computação em nuvem se desenvolveu como um novo paradigma de hospedagem e fornecimento de serviços pela Internet. A indústria considera a nuvem como uma das melhores opções para reduzir os custos indiretos de TI (Tecnologia da Informação), planejando e fornecendo benefícios que aumentam em escala, conforme a demanda cresce [Farias et al. 2016]. As principais vantagens dessa estrutura são: escalabilidade; flexibilidade e a economia de capital e custos operacionais, já que o ambiente de computação utiliza Data Centers centralizados para o compartilhamento de recursos [Dhirani et al. 2018]. 
A computação em nuvem é responsável pelo fornecimento de um Pool compartilhado de recursos que podem ser rapidamente acessados com o mínimo esforço de gerenciamento [Mell and Grance 2016]. Seus serviços de implementação e manutenção são recursos apresentados de forma transparente ao usuário, sendo amplamente categorizados como: IaaS (Infrastructure as a Service); PaaS (Platform as a Service) e SaaS (Software as a Service) [Nivetha and Vijayakumar 2016].

O cenário de nuvem vem testemunhando um crescimento sem precedentes nos últimos anos, devido à sua flexibilidade, motivando muitos negócios relacionados à hospedagem de recursos em nuvem a adotarem este modelo [Pham et al. 2016]. Os Data Centers basicamente são implantados para atender às necessidades de computação demandada pelos requisitantes [Monil and Malony 2017]. Os grandes investimentos estão relacionados também com estudos de viabilidade realizados por CSP (Amazon, Azure e Google), visando minimizar o risco em investimentos. Segundo a Dell, 8 em cada 10 empresas investirão na nuvem até 2020 [Dell 2018].

Avaliar o processo de implantação de infraestruturas é um procedimento dispendioso, demandando intensa análise, que impacta tanto no meio acadêmico quanto na indústria. Embora muito se discuta sobre TCO, CAPEX (Capital Expenditure), OPEX (Operational Expenditure), ROI (Return on Investment), há âmbitos pouco explorados na literatura. Dessa forma, deve-se considerar a larga utilização de infraestruturas de TI pela nuvem sob amplos aspectos, tais como: a relação custo-benefício; resfriamento; localização e desempenho dos data centers[Fang et al. 2017].

Uma análise econômica da nuvem como um modelo de negócio é feita em [Filiopoulou et al. 2015], onde são apresentados os parâmetros de maior impacto no custo de implantação da nuvem, montando um cenário de apoio à tomada de decisão. Já em [Sharma et al. 2012], um modelo de nuvem é simulado, avaliando os custos de nuvem com diferentes opções de serviços, concluindo que os parâmetros operacionais impactam no modelo econômico-financeiro da nuvem. Em [Kozhipurath 2012] um modelo de implantação orientado a custo para plataformas de serviços em nuvem é apresentado. Uma formulação matemática é proposta, contrapondo o estabelecimento de serviços aos fornecidos pelo provedor.

Este trabalho sintetiza as questões relacionadas aos custos em nuvem através de um modelo baseado em: relação de equipamentos e componentes de infraestrutura; projeções de custo de implantação e operação de data centers. Na literatura há poucos relatos dos parâmetros utilizados para a montagem dos cenários em nuvem, como ROI, eficiência dos data centers, questões relacionadas ao custo-benefício e sobretudo, uma comparação das estruturas disponíveis no mercado. O objetivo é fornecer um aparato técnico-financeiro para que possam ser previstos investimentos e riscos envolvidos no processo operação de infraestruturas, além de preencher as lacunas da literatura científica.

O restante deste artigo está organizado da seguinte forma: a seção 2 apresenta o modelo de custo proposto. A seção 3 apresenta o cenário de avaliação, enquanto os resultados e discussões acerca deste trabalho estão na seção 4. Por fim, a seção 5 contém conclusão do trabalho. 


\section{Modelo de Custo Proposto}

Avaliar um cenário de nuvem é uma tarefa complexa, pois o mercado é dinâmico em se tratando de investimentos de infraestruturas. O modelo de avaliação é baseado em métodos amplamente disseminados no contexto de investimentos, como é visto na Figura 1 (TCO, CAPEX e OPEX). Estes métodos são formas eficientes de se validar o contexto de implantação de data centers, um vez abordados todos os parâmetros pertinentes a computação em nuvem.

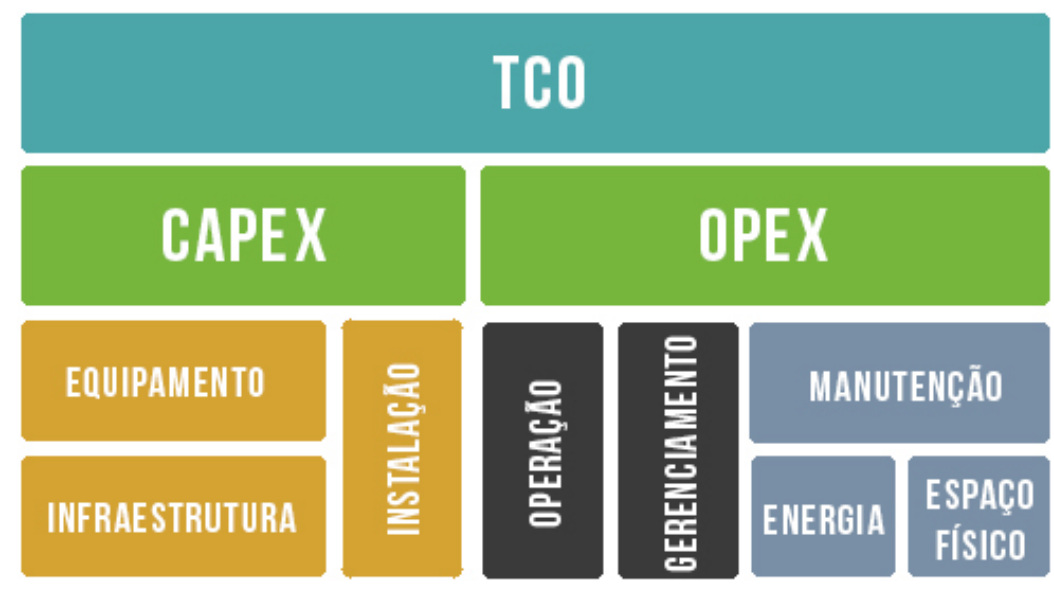

Figura 1. Modelo de Custo Total de Propriedade.

O TCO exibido na Figura 1, agrega os principais parâmetros de avaliação, como o CAPEX, relacionado às despesas de capital (equipamentos, infraestrutura e instalação) e o OPEX relativo aos custos de manutenção (operação, gerenciamento, manutenção, energia e espaço físico).

\subsection{Desagregação do TCO}

O termo desagregação se refere à fragmentação e análise de um modelo de avaliação [Cui et al. 2017], como o TCO, dividido em CAPEX e OPEX. Essa metodologia se baseia nos principais elementos de infraestruturas em relação a aquisição de bens e serviços.

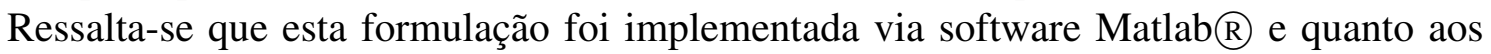
parâmetros, o valor foi estimado em US\$ conforme retratado na literatura.

\subsection{Capital Expenditure}

O CAPEX é calculado através dos custos de investimentos iniciais, como: aquisição de equipamentos de infraestrutura (servidores, cabeamento, dispositivos de rede, computadores entre outros) e custo de instalação (custo de mão de obra e de instalação).

$$
C A P E X=\sum_{i=1}^{n}\left(C_{\text {Device }}+C_{\text {inst }}\right)
$$

O CAPEX (Equação 1) pode ser calculado através do somatório de $\sum$, o qual é constituído pela soma do custo de aquisição de equipamentos $(C$ device) e de instalação (Cinst). 


\subsubsection{Aquisição de Equipamentos}

Este custo representa as taxas de aquisição de componentes físicos de uma infraestrutura, como por exemplo, servidor, rack, switch, roteador, fonte de alimentação, cabeamento, equipamento de resfriamento, móveis e utensílios para o ambiente entre outros [Kozhipurath 2012] (Equação 2).

$$
C_{\text {device }}=n_{h} * c_{h}+n_{c} * c_{c}+n_{r} * c_{r}+n_{n e t} * c_{n e t}+n_{d} * c_{d}
$$

Para implantar uma infraestrutura computacional com características típicas de nuvem, investe-se em: número de servidores $(n h)$, número de equipamentos de refrigeração $(n c)$, número de racks $(n r)$, número de dispositivos de rede (nnet), número de acessórios e utensílios $(n d)$.

\subsubsection{Infraestrutura}

O cálculo de custo de infraestrutura com as despesas para instalação de equipamentos, taxas de mão de obra por este serviço e sobretudo, implantação dos componentes são expresso na Equação 3.

$$
C_{\text {inst }}=N_{\text {nodecount }} * C_{w}+T_{h w}
$$

De acordo com [Lähteenmäki et al. 2016], o custo de infraestrutura (Equação 3), ou instalação, é relacionado ao $N$ nodecount (quantidade de data centers $C_{w}$ como o preço de diária por trabalho especializado) e $T_{h w}$ como tempo necessário para instalação.

\subsection{Operational Expenditure}

O OPEX representa as despesas operacionais de equipamentos, especificando todos os custos periódicos de infraestrutura em seu período de funcionamento [Rahman et al. 2013]. Esta formulação é concebida através dos custos de energia, gerenciamento, resfriamento, manutenção e aluguel de espaço físico. Este ativo aumenta exponencialmente conforme os ativos são acrescidos aos custos de infraestruturas conforme visto na Equação 4.

$$
\text { OPEX }=\sum_{i=1}^{n}\left(C_{\text {conf }}+C_{\text {swops }}+C_{\text {power }}+C_{\text {cooling }}+C_{s}+C_{d}\right)
$$

O OPEX é constituído pelo somatório $(i=1)$ expresso por $\sum$ de $C_{\text {conf }}$ (gerenciamento de configuração), $C_{\text {swops }}$ (Atividade de operação de softwares do datacenter), $C_{\text {power }}$ (custo de energia), $C_{\text {cooling }}$ (Resfriamento), $C_{s}$ (espaço físico) e $C_{d}$ (depreciação). Há um acréscimo anual de 1,03 sob o acumulado, isso vale para os três elementos do OPEX (energia, resfriamento e mão de obra). Este incremento refere-se ao ajuste anual do OPEX, a contar a partir do segundo ano de funcionamento da infraestrutura [Farias et al. 2016].

\subsubsection{Gerenciamento}

Este custo pertence ao gerenciamento de configuração realizado na infraestrutura, cuja licença é adquirida de acordo com a quantidade de servidores (Equação 5).

$$
C_{\text {conf }}=N_{\text {nodecount }} * N_{t}+C_{c m}
$$


Conforme citado em [Lähteenmäki et al. 2016], o gerenciamento é especificado de acordo com a quantidade de data centers $\left(N_{\text {nodecount }}\right)$, a quantidade de servidores $\left(N_{t}\right)$ da infraestrutura e o custo de gerenciamento $\left(C_{c m}\right)$ por unidade de software.

\subsubsection{Operação}

A operação representa os custos com licenças de softwares destinados aos sistemas de virtualização e gerenciamento de infraestrutura. Embora seja específico, o custo de operação detém grande impacto para avaliação do TCO, uma vez considerados vários sistemas de gerenciamento.

$$
C_{\text {swops }}=N_{\text {nodecount }} * N_{t}+C_{c l}
$$

A Equação 6 é baseada em: quantidade de data centers (Nnodecount); quantidade de servidores $(N t)$ e o custo médio de suporte $\left(C_{c l}\right)$, pois estes são componentes operacionais de uma infraestrutura em nuvem [Lähteenmäki et al. 2016].

\subsubsection{Custo de Energia}

O custo de energia é considerado o maior ativo do OPEX, pois representa o consumo energético de todos os componentes físicos, como a rede, dispositivos, condutores, entre outros componentes dessa natureza.

$$
C_{\text {power }}=\left(C_{e-k w h} * 30 * 24\right) * \operatorname{PUE}\left(S P U E *\left(P_{\text {server }}+P_{\text {net }}\right)\right)
$$

A Equação 7 especifica o valor médio cobrado por $k W h$ em Dólar (USD) em $C_{e-k W h}$ no período equivalente a 1 mês de funcionamento do data center, segundo [Cui et al. 2017] e [Hardy et al. 2013]. O PUE representa a eficácia no uso de energia, enquanto o $S P U E$ é a eficácia do servidor em específico, para ambos, quanto mais próximo de 1, mais eficientes são os componentes. Os ativos de Pserver e Pnet representam o consumo energético dos servidores. Os valores mais utilizados para estimar o grau de eficiência de infraestruturas de TI está entre 1,3 e 2, conforme visto em [Ajeh et al. 2014], [Lähteenmäki et al. 2016].

\subsubsection{Resfriamento}

O custo de resfriamento refere-se às taxas recorrentes dos componentes responsáveis por manter a temperatura do data center estável, evitando altas temperaturas que podem acarretar em maior consumo energético. $\mathrm{O}$ consumo energético dos componentes de resfriamento geralmente correspondem a cerca de $25 \%$ a $50 \%$ do consumo total de energia do data center [Iyengar et al. 2012].

$$
C_{\text {cooling }}=\left(S P U E * P_{\text {server }}+P_{\text {net }}\right) * C_{c p, W}
$$

São utilizados os $S P U E$ como a eficácia no uso de energia do servidor, o consumo de energia do servidor $\left(P_{\text {server }}\right)$, a potência total da rede $\left(P_{\text {net }}\right)$ e o custo de aquisição da infraestrutura de resfriamento $\left(C_{c p, W}\right)$ [Ajeh et al. 2014]. Este ativo considera o consumo médio mensal dos equipamentos de refrigeração, incluindo a taxa de aumento de 1,03 no valor acumulado após o primeiro ano. 
Com os grandes sistemas de refrigeração predominantes no cenário de infraestruturas de TI, nota-se que seu custo carrega uma parcela significativa do consumo total de energia e pode variar de acordo com a escalabilidade do data center.

\subsubsection{Espaço Físico}

O custo de espaço físico é o valor do aluguel do espaço onde será implantada a infraestrutura. É necessário um ambiente que respeite as mínimas características de um data center, com espaço suficiente para instalação de todos os componentes. O valor é especificado por metro quadrado, sendo esta a forma mais convencional (Equação 9).

$$
C_{s}=P * T_{m^{2}} * M
$$

O custo de aluguel com espaço físico trabalha com o custo médio por metro quadrado $(P)$, tamanho médio do espaço em metros quadrados $\left(T_{m^{2}}\right)$ e a quantidade de meses $(M)$ no qual é baseada a avaliação de custo [Ajeh et al. 2014].

\subsubsection{Custo de Manutenção}

Estes custos representam os gastos relacionados à manutenção de infraestrutura, aglutinando os gastos com energia e aluguel de espaço físico, sendo estes, valores que se repetem a cada período de cobrança (Equação 10).

$$
C_{m}=C_{s}+C_{\text {power }}+C_{\text {cooling }}
$$

A Equação 11 corresponde ao cálculo de manutenção da infraestrutura representado pelo custo de aluguel do espaço físico $\left(C_{s}\right)$, Custo de energia $\left(C_{\text {power }}\right)$ e pelo custo de resfriamento $\left(C_{\text {cooling }}\right)$. A manutenção é outro aspecto operacional, considerando os ativos que mantém seu bom funcionamento. Estes custos são estabelecidos somando todos os ativos recorrentes, como os valores incorridos mensalmente.

\subsubsection{Retorno Sobre o Investimento}

O ROI é um indicador do negócio responsável por apresentar características de recuperação de valor, ou seja, lucratividade. No contexto de infraestrutura de nuvem, o ROI especifica a porcentagem de recuperação de um investimento, consistindo em uma modalidade análoga à rentabilidade dos provedores de serviço de nuvem.

$$
R O I(\%)=\frac{\text { NetProjectBenefits }}{\text { ProjectCosts }} * 100
$$

O ROI relaciona os benefícios líquidos do projeto (NetProjectBenefits) aos custos do projeto (ProjectCosts). A razão é geralmente expressa como porcentagem, quando os valores fracionários são multiplicados por 100 [Mateljan et al. 2010]. A equação é bastante utilizada para medir os rendimentos obtidos a partir de recursos investidos, mostrando se houve ou não prejuízos em um plano de investimento.

Há uma outra abordagem complementar ao ROI, é o PRI (Prazo de Retorno Investimento), sendo este um indicador de atratividade do negócio, pois mostra o tempo necessário para que se recupere todo o investimento [Pires et al. 2015]. O PRI foca-se em determinar o ponto de equilíbrio entre a curva do TCO e a de lucratividades, mostrando projeções expressivas. 
Tabela 1. Parâmetros de Implementação de Infraestrutura.

\begin{tabular}{|c|c|c|}
\hline Equipamento / Serviço & Qtd & Valor Normalizado (US/\$) \\
\hline Servidor & 13 & 10000 \\
\hline Equipamento de Ressfriamento & 13 & 900 \\
\hline Rack's & 2 & 800 \\
\hline Dispositivo de Rede & 5 & 1000 \\
\hline Custo de Energia (Kwh) & 1 & 0.65 \\
\hline PUE & 1 & 1.7 \\
\hline SPUE & 1 & 1.4 \\
\hline Licença (S/H) & 13 & 3339 \\
\hline Trabalho Especializado (S/H) & 1 & 3339 \\
\hline Moveis e Utensilio & 2 & 2000 \\
\hline Consumo de Energia do Servidor (W) & 1 & 400 \\
\hline
\end{tabular}

\section{Cenário de Avaliação Proposto}

A proposta apresentada considera um centro de dados genérico que consiste em uma infraestrutura provisionada por um conjunto de hardware/software destinados ao fornecimento de serviços (rede, armazenamento, processamento, serviços e aplicativos). Esta infraestrutura é composta por um conjunto de componentes físicos, visando obter resultados conclusivos sobre a implantação do ambiente computacional.

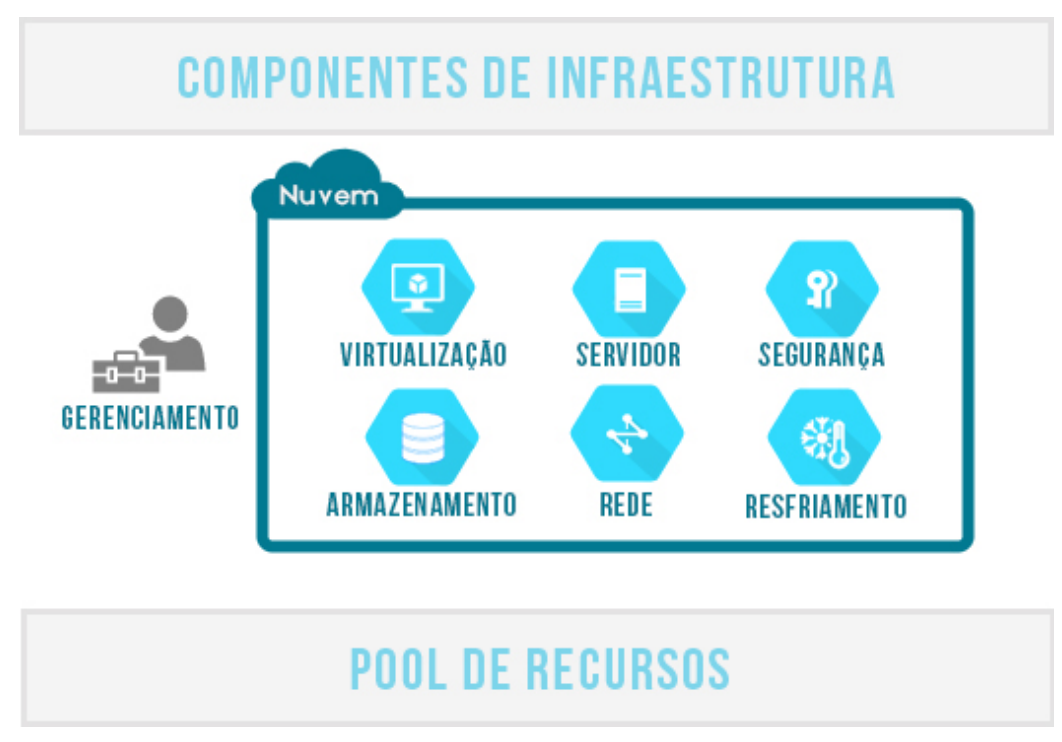

Figura 2. Modelo de Infraestrutura e componentes em nuvem.

Um modelo de arquitetura é apresentado pela Figura 2, contendo características conforme os métodos utilizados no ramo de avaliação de custos, composto por diferentes tipo de tecnologias e equipamentos. Nesta ilustração, são mostrados todos os componentes, englobando uma camada mais interna de elementos de virtualização, armazenamento, servidores, resfriamento, segurança e de rede. Os elementos (Tabela 1) ilustram um modelo de infraestrutura de TI, cuja finalidade é o fornecimento escalável e flexível de recursos, utiliza o conceito de Pool de recursos tal qual é provisionados pelos CSP.

Para este cenário de infraestrutura, são considerados 5 anos de depreciação de equipamentos, equivalente a desvalorização de $20 \%$ ao ano [Farias et al. 2016] 


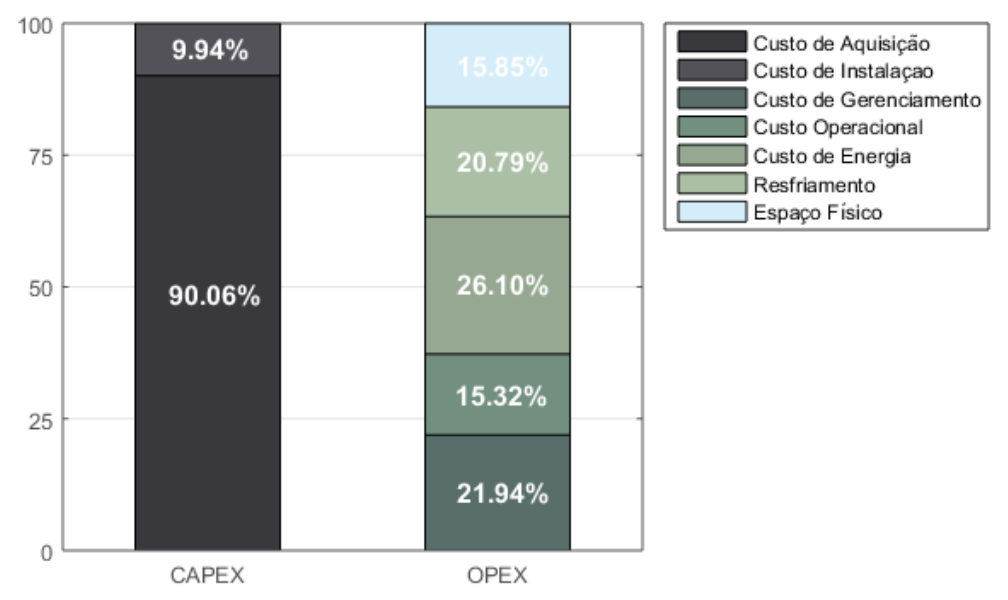

Figura 3. Custo Total de Propriedade de Infraestrutura.

[Pires et al. 2015]. Os parâmetros (Tabela 1) foram obtidos de [Barroso et al. 2013], [Kozhipurath 2012], [Cui et al. 2017], [Hardy et al. 2013], [Ajeh et al. 2014] e [Lähteenmäki et al. 2016], apresentando os valores de cada componente. Sua organização consiste em:equipamentos e serviços, quantidade e o valor médio em Dólar.

\section{Resultados e Discussões}

A principal estratégia empregada foi analisar o TCO por partes, que varia desde a aquisição, operação e manutenção de equipamentos dos data centers. Dá-se maior ênfase aos custos de maior impacto do TCO (energia, mão de obra e aluguel de espaço físico) que estão sujeitos a um acréscimo anual e consequentemente, estão em constante crescimento. Para ilustrar o cenário de investimentos de capital e operacional, a Figura 3 a seguir apresenta os custos mais significativos de implantação de um data center em nuvem.

A proporção de cada elemento do TCO indica quais componentes são mais sensíveis aos investimentos, como o aquisição com $90.06 \%$ e instalação com $9.94 \%$ em relação aos US\$ 167,996.00 de CAPEX. Já o OPEX contém maior segmentação quanto ao custo de gerenciamento com $21.94 \%$, de operação com $15.32 \%$, de energia com $26.10 \%$, de resfriamento com $20.79 \%$ e o custo de espaço físico representando $15.85 \%$ do OPEX. Esta é a representação para 1 ano de investimentos, onde o capital inicial foi de US\$ $151,300.00$ e o operacional foi de US\$ 85,320.00.

Outra contribuição é a projeção de custos do TCO, onde pode-se observar o desempenho das métricas de avaliação, especialmente quando há interferência de fatores, como a taxa de crescimento anual por exemplo. A Figura 4 a seguir exemplifica esse cenário de projeção, apresentando a performance de crescimento do CAPEX, do OPEX e a taxa de variação decorrente ao longo de 10 anos.

Com a projeção expressa pela Figura 4, nota-se um comportamento decrescente das despesas de capital inicial, reduzindo de $90.06 \%$ do valor inicial de US\$167,995.00 para US\$ 16,695.00 em 10 anos, que por sua vez, essa deterioração se sucedeu devido o período de depreciação de 5 anos conforme visto em [Ajeh et al. 2014]. Após o período de depreciação, o valor do CAPEX é estabilizado por conta do custo dos equipamentos 


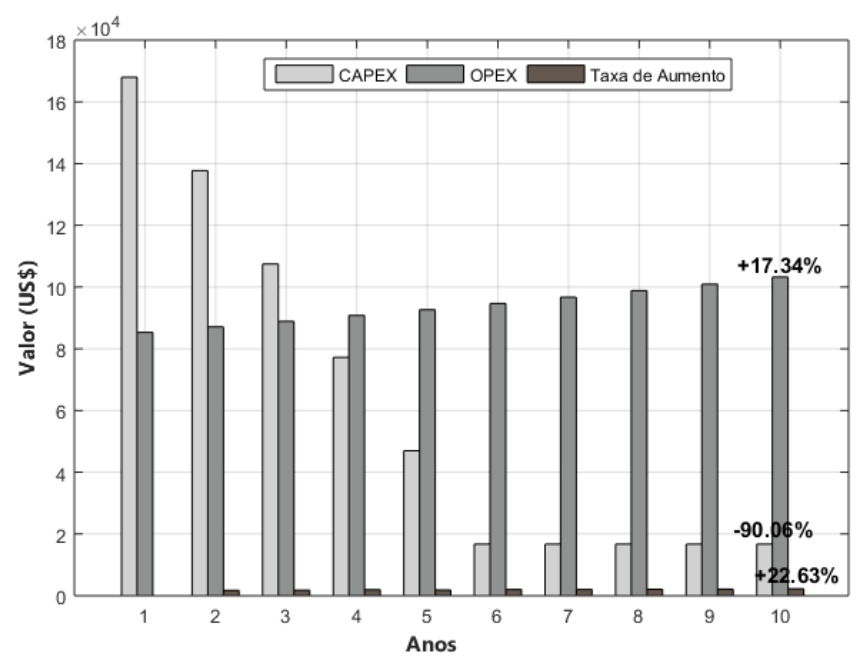

Figura 4. Projeção de TCO para 10 anos.

estarem totalmente depreciados, quando as despesas com instalação permanecem inalteradas visto que não é exposto a nenhuma variação de custos.

Nota-se um crescimento acentuado a partir do segundo ano, no qual é acrescido uma taxa de 1,03 no energia, resfriamento e na mão de obra de profissionais. Esse valor é de $17,34 \%$ ao final da projeção e equivalente a US\$103,222.49. Para 10 anos, essa taxa de acréscimo equivale a 22,63\%, correspondendo a US\$227,611.00. Em segunda análise, o fator lucratividade entra no cenário de avaliação do TCO, mostrando sua importância e o seu impacto nos investimentos a longo prazo. Esse ambiente se baseia em $275 \mathrm{MVs}$ (Máquinas Virtuais), distribuídas entre os 11 servidores com um custo médio de US\$ 56.66 por mês conforme visto na Amazon, Google Platform e Microsoft Azure.

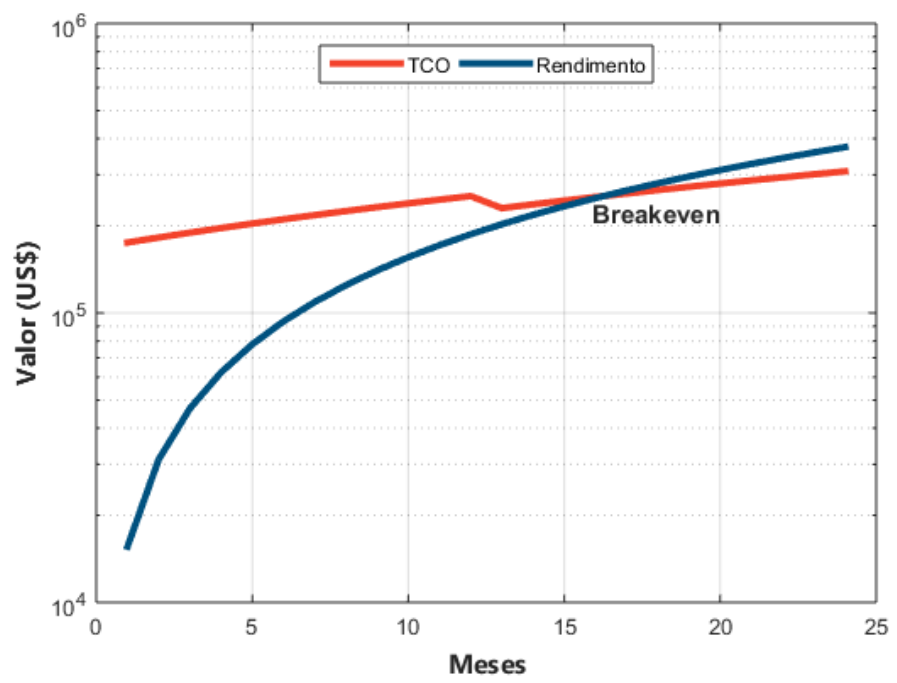

Figura 5. Prazo do retorno de investimento (PRI).

A performance de crescimento do TCO foi também analisada com base no PRI, onde a relação "investimento vs lucratividade" bem como a depreciação equipamentos podem ser visualizadas na Figura 5. A depreciação é um atributo característico de in- 
fraestruturas e, ainda que esta atue sob o TCO, o PRI é estimado comprovando que os investimentos em infraestruturas são retornados em um determinado período.

O PRI (Figura 5) compara dois fatores ao custos de infraestruturas no período de 25 meses. O rendimento foi adquirido a partir da venda de MV's para diferentes finalidades.. Assim, após o período de 12 meses (eixo x), o TCO (eixo y) deprecia-se $20 \%$ em relação ao mês anterior, perdendo US\$ 30260, enquanto o rendimento continua aumentando por não ter interferências de terceiros. O rendimento no $17^{\circ}$ mês, considerando o cenário ideal de lucro, alcançou os US\$264,885.50. No $17^{\circ}$ mês de funcionamentos do data center, momento este definido como o ponto de equilíbrio, também conhecido como "Breakeven", entre a reta de investimento e lucratividade, consequentemente, infraestrutura começa a render lucros [Hung et al. 2016] conforme a Figura 6.

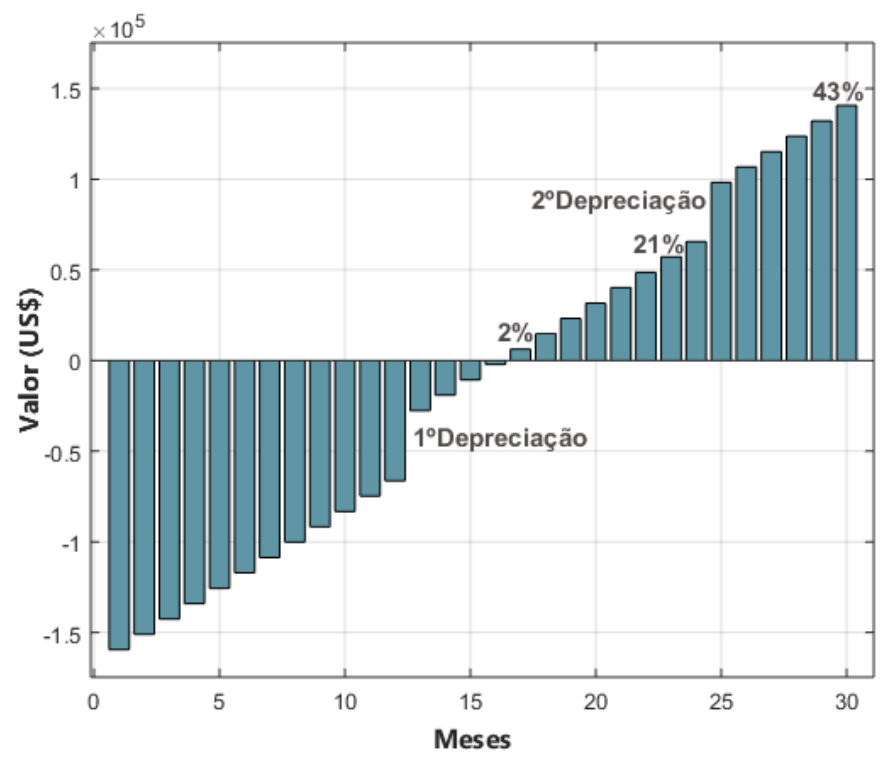

Figura 6. Retorno de Investimento (ROI).

O ROI (Figura 6) apresenta a performance de lucratividade antes e após a ocorrência do PRI, alcançando ao final dos 30 meses um rendimento de US\$ 467,445.00. $\mathrm{O}$ intervalo expressa dois comportamentos, o primeiro equivale aos 12 meses (eixo $\mathrm{x}$ ) onde a primeira depreciação de $20 \%$ ocorreu, provocando um aumento significativo no lucro e repetindo-se novamente após 24 meses, provocando então um novo aumento no lucro devido ao custo de aquisição sofrer um decréscimo de $20 \%$ no acumulado.

O ponto de equilíbrio observado na Figura 5 indica um ganho de $2 \%$ em relação ao investimento nesse período, aumentando para os $21 \%$ no momento que ocorre a segunda depreciação em 24 meses, no qual houve novamente uma variação no quesito lucro. Nesse período a depreciação estabelece uma nova ordem de crescimento para o retorno de investimento, alcançando os $43 \%$ de lucro além do valor investido no $30^{\circ}$ mês.

\section{Conclusão}

Com base nos fundamentos apresentados, percebe-se o quão imprescindível é avaliar o contexto de implantação de um data center, visto que as políticas focam cada vez mais na redução de custos e no fornecimento de serviços dinâmicos. Para tanto, este trabalho buscou agregar todos os aspectos financeiros de implantação através de uma formulação 
matemática para avaliar um cenário em nuvem, especificando os custos mais significativos de uma infraestrutura. O modelo foi representado a partir da desagregação de um TCO, com os custos de CAPEX equivalentes a US\$ 167,995.00 e os de OPEX US\$ 85,320.00 no primeiro ano.

No segundo ano, a taxa de crescimento de 1,03 entrou em vigor para custo de energia, mão de obra e resfriamento, ressaltando que estes foram os ativos mais significativos do OPEX. A deformação dos ativos operacionais é um ponto que deve ser levado em consideração, especialmente quando se visa a recuperação do ROI. Esta proposta busca futuramente ampliar os conhecimentos em modelos de custos, incluindo em seu conteúdo, aspectos já previstos pela literatura científica como análise de riscos, otimização de recursos e análise de sensibilidade no contexto de infraestruturas em nuvem.

\section{Referências}

Ajeh, D. E., Ellman, J., and Keogh, S. (2014). A Cost Modelling System for Cloud Computing. In Computational Science and Its Applications (ICCSA), 2014 14th International Conference on, pages 74-84. IEEE.

Barroso, L. A., Clidaras, J., and Hölzle, U. (2013). The Datacenter as a Computer: An Introduction to the Design of Warehouse-Scale Machines. Synthesis lectures on computer architecture, 8(3):1-154.

Cui, Y., Ingalz, C., Gao, T., and Heydari, A. (2017). Total Cost of Ownership Model for Data Center Technology Evaluation. In Thermal and Thermomechanical Phenomena in Electronic Systems (ITherm), 2017 16th IEEE Intersociety Conference on, pages 936-942. IEEE.

Dell (2018). Press Releases.

Dhirani, L. L., Newe, T., and Nizamani, S. (2018). Can IoT Escape Cloud QoS and Cost Pitfalls. In 2018 12th International Conference on Sensing Technology (ICST), pages 65-70. IEEE.

Fang, Q., Wang, J., Gong, Q., and Song, M. (2017). Thermal-Aware Energy Management of an HPC Data Center via Two-Time-Scale Control. IEEE Transactions on Industrial Informatics, 13(5):2260-2269.

Farias, F., Fiorani, M., Tombaz, S., Mahloo, M., Wosinska, L., Costa, J. C., and Monti, P. (2016). Cost-and energy-efficient backhaul options for heterogeneous mobile network deployments. Photonic Network Communications, 32(3):422-437.

Filiopoulou, E., Mitropoulou, P., Tsadimas, A., Michalakelis, C., Nikolaidou, M., and Anagnostopoulos, D. (2015). Integrating Cost Analysis in the Cloud: A SoS Approach. In Innovations in Information Technology (IIT), 2015 11th International Conference on, pages 278-283. IEEE.

Hardy, D., Kleanthous, M., Sideris, I., Saidi, A. G., Ozer, E., and Sazeides, Y. (2013). An Analytical Framework for Estimating TCO and Exploring Data Center Design Space. In Performance Analysis of Systems and Software (ISPASS), 2013 IEEE International Symposium on, pages 54-63. IEEE. 
Hung, S. N., Lee, J., and You, B.-J. (2016). Real-Time Stereo Rectification using Compressed Look-up Table with variable Breakpoint Indexing. In Industrial Electronics Society, IECON 2016-42nd Annual Conference of the IEEE, pages 4814-4819. IEEE.

Iyengar, M., David, M., Parida, P., Kamath, V., Kochuparambil, B., Graybill, D., Schultz, M., Gaynes, M., Simons, R., Schmidt, R., et al. (2012). Server Liquid Cooling with Chiller-less Data Center Design to Enable Significant Energy Savings. In Semiconductor Thermal Measurement and Management Symposium (SEMI-THERM), 2012 28th Annual IEEE, pages 212-223. IEEE.

Kozhipurath, J. (2012). Cloud Service Costing Challenges. In Cloud Computing in Emerging Markets (CCEM), 2012 IEEE International Conference on, pages 1-6. IEEE.

Lähteenmäki, J., Hämmäinen, H., Zhang, N., and Swan, M. (2016). Cost Modeling of a Network Service Provider Cloud Platform. In Cloud Engineering Workshop (IC2EW), 2016 IEEE International Conference on, pages 148-153. IEEE.

Mateljan, V., Cisic, D., and Ogrizovic, D. (2010). Cloud Database-as-a-Service (daas)ROI. In MIPRO, 2010 proceedings of the 33rd International convention, pages 11851188. IEEE.

Mell, P. and Grance, T. (2016). The NIST Definition of Cloud Computing. Special Publication 800-145 (2011). Google Scholar.

Monil, M. A. H. and Malony, A. D. (2017). QoS-Aware Virtual Machine Consolidation in Cloud Datacenter. In Cloud Engineering (IC2E), 2017 IEEE International Conference on, pages 81-87. IEEE.

Nivetha, N. and Vijayakumar, D. (2016). Modeling Fuzzy based Replication Strategy to Improve data Availabiity in Cloud Datacenter. In Computing Technologies and Intelligent Data Engineering (ICCTIDE), International Conference on, pages 1-6. IEEE.

Pham, C., Tran, N. H., Nguyen, M. N., Ren, S., Saad, W., and Hong, C. S. (2016). Hosting Virtual Machines on a Cloud Datacenter: a Matching Theoretic Approach. In Network Operations and Management Symposium (NOMS), 2016 IEEE/IFIP, pages 659-664. IEEE.

Pires, V. A. V., da Silva, M. L., Silva, C. M., Rezende, A. A. P., Cordeiro, S. A., Jacovine, L. A. G., and Soares, N. S. (2015). Economic Viability for Implantation of an Integrated Unit of Management of Solid Residuals in the Furniture Industry of ubá, MG. Cerne, 14(4):295-303.

Rahman, M., Despins, C., and Affes, S. (2013). Analysis of CAPEX and OPEX Benefits of Wireless Access Virtualization. In Communications Workshops (ICC), 2013 IEEE International Conference on, pages 436-440. IEEE.

Sharma, B., Thulasiram, R. K., Thulasiraman, P., Garg, S. K., and Buyya, R. (2012). Pricing Cloud Compute Commodities: A Novel Financial Economic Model. In Cluster, Cloud and Grid Computing (CCGrid), 2012 12th IEEE/ACM International Symposium on, pages 451-457. IEEE. 